The Astrophysical Journal, 547:L41-44, 2001 January 20

(C) 2001. The American Astronomical Society. All rights reserved. Printed in U.S.A.

\title{
UPPER LIMITS ON PERIODIC, PULSED RADIO EMISSION FROM THE X-RAY POINT SOURCE IN CASSIOPEIA A
}

\author{
M. A. Mclaughlin, ${ }^{1}$ J. M. Cordes, ${ }^{1}$ A. A. Deshpande, ${ }^{2,3}$ B. M. Gaensler,${ }^{4,5}$ \\ T. H. Hankins, ${ }^{2}$ V. M. Kaspi, ${ }^{6,7}$ AND J. S. Kern ${ }^{2}$ \\ Received 2000 October 17; accepted 2000 November 8; published 2001 January 17
}

\begin{abstract}
The Chandra X-Ray Observatory recently discovered an X-ray point source near the center of Cassiopeia A, the youngest known Galactic supernova remnant. We have conducted a sensitive search for radio pulsations from this source with the Very Large Array, taking advantage of the high angular resolution of the array to resolve out the emission from the remnant itself. No convincing signatures of a dispersed, periodic source or of isolated dispersed pulses were found, whether for an isolated or a binary source. We derive upper limits of 30 and $1.3 \mathrm{mJy}$ at 327 and $1435 \mathrm{MHz}$ for its phase-averaged pulsed flux density. The corresponding luminosity limits are lower than those for any known radio pulsar with an age less than $10^{4} \mathrm{yr}$. Our search sensitivities to single pulses were 25 and $1.0 \mathrm{Jy}$ at 327 and $1435 \mathrm{MHz}$. For comparison, the Crab pulsar emits roughly 80 pulses per minute with flux densities greater than $100 \mathrm{Jy}$ at $327 \mathrm{MHz}$ and eight pulses per minute with flux densities greater than $50 \mathrm{Jy}$ at $1435 \mathrm{MHz}$. These limits suggest that Cas A belongs to the growing population of young neutron stars that are radio-quiet.
\end{abstract}

Subject headings: pulsars: individual (Cassiopeia A) — supernova remnants

\section{INTRODUCTION}

Cassiopeia A, with an age of only $320 \mathrm{yr}$, is the youngest known Galactic supernova remnant (SNR). The supernova that gave rise to this remnant was probably discovered in 1680 , when Flamsteed (1725) observed a sixth magnitude star within $13^{\prime}$ of Cas A's location (Ashworth 1980). Because its abundances of heavy elements are consistent with those expected from the explosion of a massive star (Hughes et al. 2000), Cas A is thought to be the remnant of a Type II supernova. Given the stellar initial mass function, a large fraction of Type II supernovae are expected to leave behind neutron stars. While many searches at various wavelengths for the compact remnant in Cas A have been undertaken, only upper limits had been established until the recent Chandra discovery of an X-ray point source (XPS) near the center of the remnant (Tananbaum 1999). This XPS was later confirmed in archival ROSAT (Aschenbach 1999) and Einstein (Pavlov \& Zavlin 1999) images. The XPS is within $5^{\prime \prime}$ of the expansion center of the remnant (van den Bergh \& Kamper 1983). For a remnant distance of $3.4_{-0.1}^{+0.3} \mathrm{kpc}$, calculated through radial velocity, proper motion, and age data (Reed et al. 1995), this offset corresponds to a maximum transverse velocity of roughly $200 \mathrm{~km} \mathrm{~s}^{-1}$, a typical velocity for young pulsars (Cordes \& Chernoff 1998).

Pavlov et al. (2000) find that the X-ray spectrum of the XPS is fit well by a power law with index 2.6-4.1, with luminosity $(2-60) \times 10^{34} \mathrm{ergs} \mathrm{s}^{-1}$. Similarly, Chakrabarty et al. (2001) estimate a power-law index of 2.8-3.6 and luminosity of $(2-16) \times 10^{34}$ ergs $\mathrm{s}^{-1}$. These indices are much steeper than the X-ray spectra of active galactic nuclei (AGNs). Further-

\footnotetext{
${ }^{1}$ Astronomy Department, Cornell University, 512 Space Sciences Building, Ithaca, NY 14853.

${ }^{2}$ Physics Department, New Mexico Institute of Mining and Technology, Socorro, NM 87801.

${ }^{3}$ Raman Research Institute, Bangalore 560080, India.

${ }^{4}$ Hubble Fellow.

${ }^{5}$ Center for Space Research, Massachusetts Institute of Technology, Cambridge, MA 02139.

${ }^{6}$ Physics Department, McGill University, Montreal H3A 2T8, Canada.

${ }^{7}$ On leave from the Physics Department and Center for Space Research, Massachusetts Institute of Technology, Cambridge, MA 02139.
}

more, given the measured surface density of AGNs, the probability of finding an AGN that close to the center of the SNR is negligible. For these reasons, Pavlov et al. (2000) conclude that the XPS is almost certainly associated with the Cas A remnant. The fitted power-law indices for the XPS are also much steeper than those measured for the six young X-ray pulsars having ages less than $10^{4} \mathrm{yr}$, whose spectral indices range from 1.1 to 1.7 (Chakrabarty et al. 2001). In addition, the estimated XPS luminosities are only marginally consistent with measured young pulsar X-ray luminosities, which range from $3 \times 10^{35}$ to $4 \times 10^{36} \mathrm{ergs} \mathrm{s}^{-1}$. It is also significant that, unlike five of the six young pulsars with ages less than $10^{4} \mathrm{yr}$, Cas A shows no evidence for a synchrotron nebula surrounding the point source.

While there is much evidence for and against the neutron star nature of the XPS, the definitive proof would be the detection of pulsations. No X-ray pulsations have been found in data taken with the Chandra high-resolution camera. However, a time-tagging problem caused the timing accuracy to be degraded from $16 \mu \mathrm{s}$ to $\approx 4 \mathrm{~ms}$, allowing searches for only broad pulses with periods greater than $20 \mathrm{~ms}$ (Chakrabarty et al. 2001). The detection of radio pulsations, however, would be just as conclusive. For this reason, we have conducted a search for radio pulsations from the XPS using the Very Large Array (VLA) at frequencies of 327 and $1435 \mathrm{MHz}$. There have been several previous searches for a radio pulsar in Cas A (Davies \& Large 1970; Seiradakis \& Graham 1980; Woan \& DuffettSmith 1993; Lorimer, Lyne, \& Camilo 1998). Our VLA search is many times more sensitive, as having an accurate position for the XPS allows us to resolve out the strong emission from the remnant, which with 327 and $1435 \mathrm{MHz}$ flux densities of 6400 and $2000 \mathrm{Jy}$ is one of the strongest radio sources known.

\section{DATA}

We observed the XPS on 3 days (1999 October 23, October 31 , and November 28) with the phased-array VLA in the B configuration, where the maximum baseline is $11.4 \mathrm{~km}$. The J2000 coordinates of the XPS are R.A. $=23^{\mathrm{h}} 23^{\mathrm{m}} 27^{\mathrm{s}} .94$, decl. $=+58^{\circ} 48^{\prime} 42^{\prime \prime}$, with positional uncertainty of $1^{\prime \prime}$ (Tananbaum 1999). As the 327 and $1435 \mathrm{MHz}$ synthesized beam- 
widths are approximately $20^{\prime \prime}$ and $4^{\prime \prime}$, respectively, one beam at each frequency was sufficient for this search. During each session, the XPS was observed at center frequencies of 327 and $1435 \mathrm{MHz}$, resulting in three pointings of length 20,40, and 50 minutes at $327 \mathrm{MHz}$ and four pointings of length 40 , 40, 45, and 50 minutes at $1435 \mathrm{MHz}$. During each observing session, we observed the known pulsar PSR B0355+54, which has a period of $154 \mathrm{~ms}$ and a dispersion measure (DM) of $57 \mathrm{pc} \mathrm{cm}^{-3}$. A blank-sky pointing and the flux and phase calibrator J2350+646 (with flux densities of 27 and 5 Jy at 327 and

$1435 \mathrm{MHz}$ ) were also observed.

We used the VLA's High Time Resolution Processor (Moffett 1997) to record dual circular polarization data at a sampling frequency of $9.22 \mathrm{kHz}$. Each scan was started on a $10 \mathrm{~s} \mathrm{tick}$, tied to the VLA's hydrogen maser, compared to Universal Time through the Global Positioning System. At $327 \mathrm{MHz}, 14 \times$ $0.125 \mathrm{MHz}$ frequency channels covered a total bandwidth of $1.75 \mathrm{MHz}$. These channels were spaced noncontiguously to avoid known interference frequencies and covered a total bandwidth range of $3 \mathrm{MHz}$. At $1435 \mathrm{MHz}, 14 \times 4 \mathrm{MHz}$ channels covered a total contiguous bandwidth of $48 \mathrm{MHz}$. (As only $50 \mathrm{MHz}$ of bandwidth is available, some channels were duplicated.) The gain in each of the 28 channels (two polarizations and 14 frequencies) was calculated from observations of the calibrator source.

\section{ANALYSIS}

For each of the seven XPS data sets, we first summed rightand left-circular polarizations and then dedispersed the data over a range of trial DMs. The Taylor \& Cordes (1993) model predicts a maximum DM of approximately $75 \mathrm{pc} \mathrm{cm}^{-3}$ in the direction of the XPS. We dedispersed the data with 60 trial DMs from 0 to $300 \mathrm{pc} \mathrm{cm}^{-3}$ to allow for an underestimate or a contribution from Cas A. These $60 \mathrm{DMs}$ were spaced nonuniformly, with greater spacing at higher DMs, where the temporal smearing due to dispersion across an individual frequency channel is larger. Each dedispersed time series was Fourier transformed with 32 Mpoint and/or 16 Mpoint fast Fourier transforms (FFTs), depending on the length of the data set. Shorter FFTs of 8 and 4 Mpoints were done to increase our sensitivity to some binary pulsars. The resulting power spectra were harmonically summed (up to a maximum of 16 harmonics) to increase our sensitivity to narrow pulses (Lyne \& Smith 1998). All features in the resulting power spectra with signalto-noise ratio above a threshold of 7 were saved as pulsar candidates. For each candidate, the corresponding dedispersed time series was folded at the period corresponding to the fundamental harmonic. These profiles were inspected, and candidate lists from different days and at different frequencies were compared. While many periodic signals were detected, they were predominantly at low DM, had noiselike pulse profiles, and did not appear on multiple epochs or at multiple frequencies, and were therefore attributed to radio frequency interference (RFI). Figure 1 shows the folded pulse profiles resulting from applying the search algorithm to PSR B0355+54.

An independent Fourier analysis of the same data, carried out at the New Mexico Institute of Mining and Technology, was also unsuccessful. This analysis was sensitive to periods $\geq 10 \mathrm{~ms}$ and covered a smaller DM range but with a finer DM spacing. Three filter-bank channels with high levels of RFI were rejected, decreasing the number of spurious candidates significantly with only a $12 \%$ sensitivity loss. Power spectra were

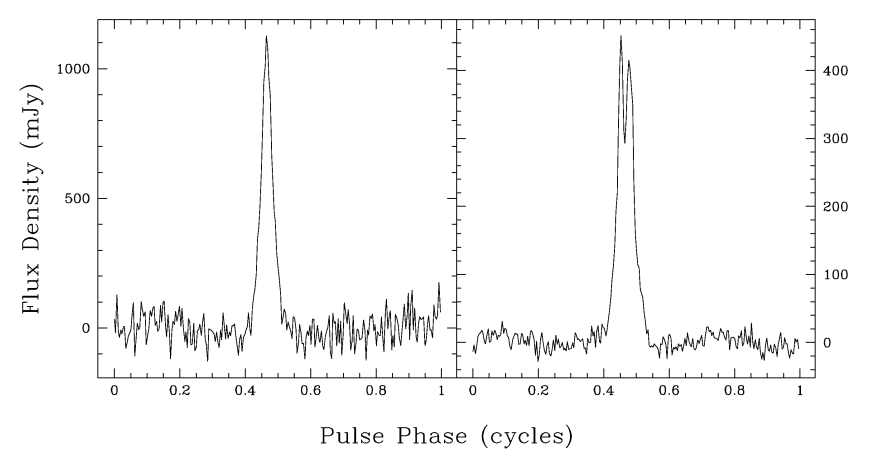

FIG. 1.-Folded pulse profiles for PSR B0355+54, which has a period of $156 \mathrm{~ms}$ and DM of $57 \mathrm{pc} \mathrm{cm}^{-3}$ at $327 \mathrm{MHz}$ (left) and $1435 \mathrm{MHz}$ (right). Note that these profiles are for 5 and 2 minutes of data at 327 and $1435 \mathrm{MHz}$, whereas our longest XPS search scans were 50 minutes at these same frequencies.

harmonically summed up to a maximum of 32 harmonics. Final pulsar candidates were evaluated by comparing average profiles from two halves of the bandpass and two halves of profiles folded over a two-period span. These were compared with the corresponding profiles for nondedispersed data.

The Crab pulsar, currently the youngest known radio pulsar, was initially detected through its "giant" pulses, not through its time-averaged pulsed emission (Staelin \& Reifenstein 1968). Giant pulses are individual pulses with amplitudes much greater than the mean pulse amplitude (Lundgren et al. 1995). As we might expect other young pulsars to behave similarly to the Crab, we also searched for aperiodic, dispersed pulses from the XPS. We recorded all pulses with amplitudes above a signalto-noise ratio threshold of 4 for each dedispersed time series, enhancing our sensitivity to broadened pulses by repeating this thresholding with different levels of time series smoothing. Given our $4 \sigma$ threshold, we were sensitive to all single pulses with flux densities greater than $25 /\left(w_{\mathrm{ms}}\right)^{1 / 2} \mathrm{Jy}$, where $w_{\mathrm{ms}}$ is the pulse width in milliseconds, at $327 \mathrm{MHz}$ and $1.0 /\left(w_{\mathrm{ms}}\right)^{1 / 2} \mathrm{Jy}$ at $1435 \mathrm{MHz}$. Our smoothing algorithm optimized the search for pulse widths up to $15 \mathrm{~ms}$. (For comparison, the widths of the Crab pulsar's giant pulses range from roughly 0.2 to $0.4 \mathrm{~ms}$.) We found no evidence for isolated pulses from the XPS. The result of this analysis, along with the result for PSR B0355+54, is shown in Figure 2. For comparison, the Crab pulsar emits roughly 80 pulses per minute with flux densities greater than $100 \mathrm{Jy}$ at $327 \mathrm{MHz}$ and eight pulses per minute with flux densities greater than $50 \mathrm{Jy}$ at $1435 \mathrm{MHz}$.

\section{RESULTS}

To calculate an upper limit for pulsed radio emission from the XPS, we must account for the excess system temperature due to the emission from Cas A itself. The Cas A remnant is $5^{\prime}$ in size (D. A. Green $2000^{8}$ ). Hence, the ratios of our beam area to the remnant area are 0.003 and 0.0002 at 327 and $1435 \mathrm{MHz}$. Given Cas A's measured flux density and spectral index (see footnote 8), we calculate remnant flux densities of 6432 and $2060 \mathrm{Jy}$ at 327 and $1435 \mathrm{MHz}$, contributing to an increase in system temperature of a modest $40 \mathrm{~K}$ at $327 \mathrm{MHz}$ and a mere $1 \mathrm{~K}$ at $1435 \mathrm{MHz}$ for the phased array. However, while the

\footnotetext{
${ }^{8}$ A Catalogue of Galactic Supernova Remnants (2000 August version; Cambridge: Mullard Radio Astronomy Observatory, Cavendish Laboratory); available at http://www.mrao.cam.ac.uk/surveys/snrs.
} 


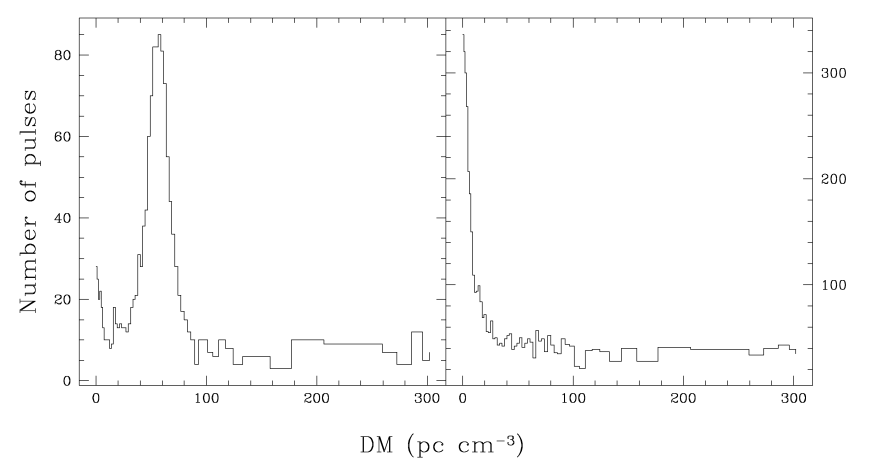

FIG. 2.-Number of isolated, dispersed pulses at $327 \mathrm{MHz}$ above a $4 \sigma$ threshold vs. DM for a 5 minute observation of PSR B0355+54 (left) and a 50 minute observation of the XPS (right). While a peak at the $57 \mathrm{pc} \mathrm{cm}^{-3}$ DM of PSR B0355+54 is obvious, there is no evidence for such an excess from the XPS. The peak at low DM in the right plot is consistent with interference and is similar to the number of counts observed in the consecutive blank-sky pointing. In the absence of RFI and/or a pulsar, we would expect a flat distribution.

phased array resolves out emission from the nebula, each single dish will see the whole remnant, leading to an increase in system temperature of $475 \mathrm{~K}$ at $327 \mathrm{MHz}$ and $230 \mathrm{~K}$ at $1435 \mathrm{MHz}$. By comparison, a single dish with the same collecting area as the VLA would suffer increases of system temperature of 13,000 and $6200 \mathrm{~K}$ at 327 and $1435 \mathrm{MHz}$.

We calculate the minimum detectable flux density of our search using the expression (Cordes \& Chernoff 1997)

$$
S_{\mathrm{min}}=\frac{\eta T_{\mathrm{sys}}}{G \sqrt{N_{\mathrm{pol}} \Delta \nu \Delta t N_{\mathrm{FFT}}}} \frac{\sqrt{N_{h}}}{\sum_{l=1}^{N_{h}} R_{l}},
$$

where $\eta$ is the signal-to-noise ratio threshold used, $T_{\text {sys }}$ is the system temperature, $G$ is the telescope gain, $N_{\text {pol }}$ is the number of polarization channels, $\Delta \nu$ is the total bandwidth, $\Delta t$ is the sample interval, $N_{\mathrm{FFT}}$ is the number of points in the FFT, $N_{h}$ is the optimal number of harmonics with which a pulsar can be detected, and $R_{l} \leq 1$ is the ratio of the $l$ th harmonic to the fundamental. The value of $N_{h}$ will depend on the pulsar's duty cycle, or ratio of pulse width to period, with narrower pulses producing more harmonics. For this calculation, we take the intrinsic duty cycle to be the minimum of 0.3 and $0.03\left(P_{\mathrm{ms}} / 1000 \text {. }\right)^{-0.5}$, where $P_{\mathrm{ms}}$ is the pulse period in milliseconds (Biggs 1990). Pulses may then be additionally broadened by propagation effects, including scattering and dispersion. The Taylor \& Cordes (1993) model predicts $1.7 \mathrm{~ms}$ and $3 \mu \mathrm{s}$ of pulse broadening due to scattering in the direction of the XPS and for a distance of $3.4 \mathrm{kpc}$ at 327 and $1435 \mathrm{MHz}$, respectively.

Figure 3 shows the dependence of our search sensitivity on $\mathrm{DM}$ and period at the two observing frequencies, accounting for the effects of scattering and dispersion on the intrinsic duty cycle of the pulsar. Assuming values for period and DM of $30 \mathrm{~ms}$ and $75 \mathrm{pc} \mathrm{cm}^{-3}$, we calculate $7 \sigma$ upper limits of 30 and $1.3 \mathrm{mJy}$ at 327 and $1435 \mathrm{MHz}$. As shown in Figure 3, these limits will decrease for longer period, smaller duty cycle, and lower DM. There are no hardware limitations to our maximum detectable period, determined solely by the length of the FFT.

While there is no infrared or optical evidence for a binary companion (van den Bergh \& Pritchet 1986), acceleration ef-

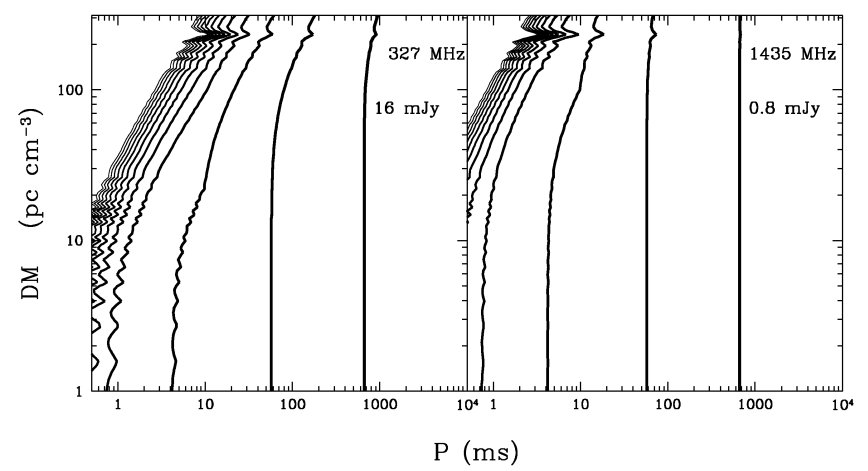

FIG. 3.-Contours of pulsed search $7 \sigma$ sensitivity vs. period and DM for searches at both 327 and $1435 \mathrm{MHz}$. The thickest (rightmost) line corresponds to a sensitivity of $16 \mathrm{mJy}$ at $327 \mathrm{MHz}$ and of $0.8 \mathrm{mJy}$ at $1435 \mathrm{MHz}$. Minimum detectable flux densities increase by a factor of 2 with each subsequently thinner line. The contours are not smooth because of the finite spacing of our trial DMs.

fects from such a companion would cause pulse smearing, decreasing our sensitivity. We estimate the signal-to-noise ratio degradation introduced for various orbital systems by taking a Gaussian pulse and applying orbital smearing by iteratively calculating emission times across the orbit. Smearing times due to time constant, DM, and scattering are calculated and applied to the orbital-smeared pulse. We calculate the best harmonic sum for the resultant waveform and compare this to the best harmonic sum for a waveform with no orbital smearing to calculate the loss in signal-to-noise ratio. For a $30 \mathrm{~ms}$ period and duty cycle of 0.2 , a 1 solar mass companion in an $8 \mathrm{hr}$ circular orbit with an inclination angle (i.e., angle between the orbital plane and our line of sight) of $30^{\circ}$, our sensitivity decreases by roughly a factor of 2 , averaging over all orbital phases, for a 16 Mpoint FFT. In the case of our 8 and 4 Mpoint FFTs, these same binary parameters would decrease our search sensitivity by factors of 2.4 and 2.8 over the original 16 Mpoint sensitivity to an unaccelerated pulsar. Although the nominal sensitivities are less for these shorter FFT lengths, the shorter FFTs would allow us to detect pulsars in fast binaries where the binary smearing during a longer FFT is comparable to or greater than the pulse period. The sensitivity would be further decreased for smaller spin periods, faster orbits, and more massive companions. If the orbit was eccentric, the net smearing, averaged over all orbital phases, would be less, and the resultant sensitivity would be higher. Accretion from a binary companion could completely quench the radio emission.

Interstellar scintillation modulations of the flux of the XPS are mostly negligible during our observations. The predicted scintillation bandwidth and timescale (see Cordes \& Rickett 1998), assuming a uniform medium and a velocity of $50 \mathrm{~km} \mathrm{~s}^{-1}$, are approximately $0.1 \mathrm{kHz}$ and $30 \mathrm{~s}$ at $327 \mathrm{MHz}$ and $0.6 \mathrm{MHz}$ and $150 \mathrm{~s}$ at $1435 \mathrm{MHz}$, in both cases too small to significantly affect our quoted upper limits. If this velocity is higher than $50 \mathrm{~km} \mathrm{~s}^{-1}$, as a young pulsar's velocity is likely to be (Cordes $\&$ Chernoff 1998), the predicted scintillation timescale will be smaller and will therefore be even less of a factor in our analysis.

We have also used VLA images of Cas A to search for a radio continuum point source at the position of the XPS. At 1.3 and $4.4 \mathrm{GHz}$, we find $5 \sigma$ upper limits on the flux density from the XPS of 40 and $6 \mathrm{mJy}$, respectively. While not as constraining as those derived from our VLA and Arecibo pulsed searches described above, these continuum upper limits are 
completely independent of the period, dispersion, and scattering of any radio pulsations from this source.

\section{CONCLUSIONS}

We have calculated upper limits of 30 and $1.3 \mathrm{mJy}$ at 327 and $1435 \mathrm{MHz}$ for the phase-averaged pulsed flux density from the XPS in Cas A. We did not detect any single, dispersed pulses above our sensitivity of 25 and $1.0 \mathrm{Jy}$ (assuming a pulse width of $1 \mathrm{~ms}$ ) at 327 and $1435 \mathrm{MHz}$. We now compare our upper limit to those from previous Cas A searches. Davies \& Large (1970) carried out an unsuccessful $408 \mathrm{MHz}$ search for single pulses from Cas A. Seiradakis \& Graham (1980) quoted a $1420 \mathrm{MHz}$ upper limit of $1.6 \mathrm{mJy}$ for pulsed emission from Cas A. However, their limit does not account for the additional system temperature contributed by the nebula, significant for their beamwidth of 9'. More recently, Woan \& Duffett-Smith (1993) published an upper limit of $80 \mathrm{mJy}$ at $408 \mathrm{MHz}$, and Lorimer et al. (1998) published an upper limit of $46 \mathrm{mJy}$ at $606 \mathrm{MHz}$. For a typical pulsar spectrum, our $1435 \mathrm{MHz}$ upper limit is an order of magnitude better than these previously published limits due to the known position of the XPS and the power of the VLA to resolve out the excess emission from the nebula.

Our upper limit of $1.3 \mathrm{mJy}$ at $1435 \mathrm{MHz}$ translates to a luminosity of $15 \mathrm{mJy} \mathrm{kpc}^{2}$ for a neutron star at a distance of $3.4 \mathrm{kpc}$. This is lower than the mean $1400 \mathrm{MHz}$ luminosity of $30 \mathrm{mJy} \mathrm{kpc}{ }^{2}$ estimated for young pulsars (Frail \& Moffett 1993) and is lower than the luminosity of any known radio pulsar with characteristic age less than $10^{4} \mathrm{yr}$ (see Figs. 1 and 2 of Brazier \& Johnston 1999). This suggests that the XPS in Cas $\mathrm{A}$ is either not a pulsar, is a radio-quiet pulsar, or is a radio pulsar beamed away from us. While evidence suggests that pulsar beams are wider at short periods (see, e.g., Biggs 1990) and that they are therefore likely to intersect the Earth's line of sight, individual pulsars show mixed properties. For instance, the Crab pulsar shows narrow radio components that may be part of a large beam, but the profile of another young pulsar in the Large Magellanic Cloud, PSR B0540-69, is very broad (Manchester et al. 1993). Furthermore, it is difficult to disentangle true beam width from viewing angle for these pulsars. It is certainly possible that, like the Geminga pulsar, the XPS shows no radio pulses but is an X-ray, and perhaps gamma- ray, pulsar. Although there is no EGRET point source near the position of the XPS, gamma-ray searches with future, more sensitive instruments, could be successful. Further measurements of the spectrum and time variability of the XPS will also help determine which of the above scenarios is the case.

This search adds to the growing body of evidence that there are manifestations of young neutron stars other than the standard Crab-like radio pulsar. Although it is widely accepted that radio pulsars are born in supernovae, fewer than $10 \%$ of searches targeted at SNRs have found young (age $<25 \mathrm{kyr}$ ) pulsars (Kaspi et al. 1996; Gorham et al. 1996; Lorimer et al. 1998). Some of this may be due to scattering at low frequencies in the Galactic plane and the decreased sensitivity due to the bright remnants, but it also hints at the unrecognized diversity of the neutron star population. While some neutron stars are detected as radio pulsars, others may be born as anomalous $\mathrm{X}$-ray pulsars, soft gamma-ray repeaters, quiescent neutron stars, or other classes of objects not yet discovered (Kaspi 2000; Gotthelf \& Vasisht 2000). The pathways to and percentages of these different classes of objects are not yet clear. However, future Chandra detections of compact X-ray sources and largescale radio pulsar surveys, like the Parkes Multibeam Survey (Lyne et al. 2000), will help to answer these questions.

We wish to thank Miller Goss for expediting our observations and Duncan Lorimer for helpful comments on the manuscript. We thank Larry Rudnick and Barron Koralesky for kindly providing their radio images of Cas A for our analysis. This work was partially supported by NSF grant AST 96-18408. The work was also supported by the National Astronomy and Ionosphere Center, which is operated by Cornell University under cooperative agreement with the National Science Foundation (NSF). The National Radio Astronomy Observatory is a facility of the NSF operated under cooperative agreement by Associated Universities, Inc. B. M. G. acknowledges the support of NASA through Hubble Fellowship grant HST-HF-01107.01-A awarded by the Space Telescope Science Institute, which is operated by the Association of Universities for Research in Astronomy, Inc., for NASA under contract NAS5-26555. V. M. K. acknowledges support from an Alfred P. Sloan research fellowship, from an NSF CAREER award (AST 98-75897), and from an NSERC research grant (RGPIN228738-00).

\section{REFERENCES}

Aschenbach, B. 1999, IAU Circ. 7249

Ashworth, W. B. 1980, J. Hist. Astron., 11, 1

Biggs, J. D. 1990, MNRAS, 245, 514

Brazier, K. T. S., \& Johnston, S. 1999, MNRAS, 305, 671

Chakrabarty, D., Pivovaroff, M. J., Hernquist, L. E., Heyl, J. S., \& Narayan, R. 2001, ApJ, in press

Cordes, J. M., \& Chernoff, D. F. 1997, ApJ, 482, 971

- 1998, ApJ, 505, 315

Cordes, J. M., \& Rickett, B. J. 1998, ApJ, 507, 846

Davies, J. G., \& Large, M. I. 1970, MNRAS, 149, 301

Flamsteed, J. 1725, in Historia Coelsetis Britannicae (London: H. Meere), in folio, DCC.f.9, DCC.f.10, DCC.f.11

Frail, D. A., \& Moffett, D. A. 1993, ApJ, 408, 637

Gorham, P., Ray, P., Anderson, S., Kulkarni, S., \& Prince, T. 1996, ApJ, 458, 257

Gotthelf, E. V., \& Vasisht, G. 2000, in IAU Colloq. 177, Pulsar Astronomy -2000 and Beyond, ed. M. Kramer, N. Wex, \& R. Wielebinski (ASP Conf. Ser. 202; San Francisco: ASP), 699

Hughes, J. P., Rakowski, C. E., Burrows, D. N., \& Slane, P. O. 2000, ApJ, 528, L109

Kaspi, V. M. 2000, in IAU Colloq. 177, Pulsar Astronomy-2000 and Beyond, ed. M. Kramer, N. Wex, \& R. Wielebinski (ASP Conf. Ser. 202; San Francisco: ASP), 485
Kaspi, V. M., Manchester, R., Johnston, S., Lyne, A., \& D'Amico, N. 1996, AJ, 111, 2028

Lorimer, D. R., Lyne, A. G., \& Camilo, F. 1998, A\&A, 331, 1002

Lundgren, S. C., et al. 1995, ApJ, 453, 433

Lyne, A. G., \& Smith, F. G. 1998, Pulsar Astronomy (2d ed.; Cambridge: Cambridge Univ. Press)

Lyne, A. G., et al. 2000, MNRAS, 312, 698

Manchester, R. N., Mar, D. P., Lyne, A. G., Kaspi, V. M., \& Johnston, S. 1993, ApJ, 403, L29

Moffett, D. A. 1997, Ph.D. thesis, New Mexico Institute of Mining and Technology

Pavlov, G. G., \& Zavlin, V. E. 1999, IAU Circ. 7270

Pavlov, G. G., Zavlin, V. E., Aschenbach, B., Trümper, J., \& Sanwal, D. 2000, ApJ, 531, L53

Reed, J. E., Hester, J. J., Fabian, A. C., \& Winkler, P. F. 1995, ApJ, 440, 706

Seiradakis, J. H., \& Graham, D. A. 1980, A\&A, 85, 353

Staelin, D. H., \& Reifenstein, E. C. 1968, Science, 162, 1481

Tananbaum, H. 1999, IAU Circ. 7246

Taylor, J. H., \& Cordes, J. M. 1993, ApJ, 411, 674

van den Bergh, S., \& Kamper, K. W. 1983, ApJ, 268, 129

van den Bergh, S., \& Pritchet, C. J. 1986, ApJ, 307, 723

Woan, G., \& Duffett-Smith, P. J. 1993, MNRAS, 260, 693 